\title{
Pure Erythroid Leukemia in Remission
}

National Cancer Institute

\section{Source}

National Cancer Institute. Pure Erythroid Leukemia in Remission. NCI Thesaurus. Code C8617.

Pure erythroid leukemia that is responding to therapy, i.e. It is not progressing and possibly regressing. 